\title{
Sinnvoller Klimaschutz oder Ablasshandel?
}

\section{Der Emissionshandel und andere Klimaschutzinstrumente decken nur einen Teil der ausgestoßenen Treibhausgase ab. Die freiwillige Kompensation von Treib- hausgasemissionen ist ein neves Geschäftsfeld, das zunehmend das öffentliche Interesse auf sich zieht. Ein sinnvoller Weg oder pure Augenwischerei?}

$\mathrm{D}$

Von Wolfgang Sterk und Maike Bunse ie Kompensation von Treibhausgasen ist aus zwei Gründen von Bedeutung. Emissionsquellen wie Haushalte, die Mehrheit der klein- und mittelständischen Betriebe, Verkehr insbesondere Flugverkehr - sowie Großveranstaltungen aller Art werden nicht oder nur unzureichend erfasst. Zudem ist es normalerweise nicht möglich, die eigenen Emissionen auf Null zu reduzieren. Die freiwillige Kompensation ist ein Ansatz, der versucht, das Prinzip des Emissionshandels auf diese Emissionen anzuwenden.

Kompensation bedeutet, eine bestimmte Menge an verursachten Treibhausgasemissionen an anderer Stelle zu vermeiden oder der Atmosphäre durch so genannte Senkenprojekte zu entziehen. Senkenprojekte sind Maßnahmen, durch die der Atmosphäre Kohlendioxid entzogen und der Kohlenstoff in wachsender Biomasse gespeichert wird, also beispielsweise Aufforstungsprojekte. Emissionsminderungsprojekte sind beispielsweise Erneuerbare Energien- oder Energieeffizienzprojekte, durch die die Nutzung fossiler Energieträger vermindert wird.

Für die Kompensationsdienstleistungen hat sich eine Reihe von professionellen Anbietern am Markt etabliert, die für ihre Kunden

- die zu kompensierende Emissionsmenge berechnen,

- ein Kompensationsprojekt durchführen, durch das die entsprechende Menge an Emissionen reduziert beziehungsweise der Atmosphäre die entsprechende Menge an Treibhausgasen entzogen wird, und

- dies durch die Ausgabe von Emissionszertifikaten oder eines anderen Nachweises an den Kunden dokumentieren.

\section{Ohne schlechtes Gewissen?}

Aus Klimaschutzsicht ist die Vermeidung der eigenen Emissionen klar ihrer Kompensation vor- zuziehen. Der Klimawissenschaft zufolge müssen die weltweiten Treibhausgasemissionen bis zur Jahrhundertmitte um die Hälfte reduziert werden, um einem Klimakollaps vorzubeugen. Da den südlichen Ländern noch ein gewisses Anwachsen ihrer Emissionen zugestanden werden muss, damit sie ihre Entwicklungsbedürfnisse befriedigen können, bedeutet dies für die Industriestaaten die Notwendigkeit, ihre Emissionen um circa 80 Prozent herunterzufahren.

Die vollständige Internalisierung der externen Kosten der Treibhausgasemissionen durch verbindliche Politikmaßnahmen wie den Emissionshandel oder die Ökosteuer, sowie sektorale und technologieorientierte Maßnahmen wie das Erneuerbare-Energien-Gesetz, bringen daher einen höheren langfristigen Klimanutzen als die Kompensation, da sie die notwendigen strukturellen Änderungen in den Industriestaaten unterstïtzen. Weiterhin führt dies auch zu tatsächlichen Reduktionen, während die Kompensation bestenfalls ein Nullsummenspiel darstellt: die verursachten Emissionen werden an einer anderen Stelle wieder eingespart, aber nicht mehr. Letztendlich werden die Emissionen also bloß um den Globus hin und her verschoben. Wenn aber die eigenen Möglichkeiten zur Reduktion von Emissionen erschöpft sind, kann die Kompensation eine gangbare ,second-best“ Option sein. Sie stellt dann eine neue Möglichkeit dar, sein Verhalten besser mit den eigenen Idealen in Einklang zu bringen. Der Teufel steckt dabei allerdings im Detail, denn die verschiedenen Angebote unterscheiden sich deutlich. Ein qualitativ schlechtes Kompensationsangebot kann letztlich sogar zu steigenden Emissionen führen, wenn nämlich der Kunde sich durch die Kompensation auf der sicheren Seite wähnt und deshalb zum Beispiel gleich noch einen weiteren Flug bucht, die vermeintlich eingekaufte Emissionsreduktion aber tatsächlich gar nicht erbracht wird.
Wie oben erwähnt, können Emissionen über Emissionsminderungs- oder aber über Senkenprojekte kompensiert werden. Während Emissionsminderungsprojekte dazu führen, dass die Atmosphäre ein für allemal von den Treibhausgasen frei gehalten wird, kann der bei Senkenprojekten gespeicherte Kohlenstoff jederzeit wieder in die Atmosphäre freigesetzt werden, etwa durch einen Waldbrand oder Schädlingsbefall. Zudem greifen Senkenprojekte nicht die Ursache der Klimaerwärmung an, nämlich die auf fossilen Rohstoffen beruhenden Energiesysteme und Infrastrukturen. Aus Klimaschutzsicht sind daher Emissionsminderungsprojekte zu bevorzugen.

Die Gegenleistung für den Kunden erfolgt normalerweise in Form von Emissionszertifikaten, die durch die durchgefuihrten Projekte generiert werden, wobei ein Zertifikat einer Tonne Kohlendioxid entspricht. Diese Zertifikate werden danach normalerweise vom Markt genommen, um zu verhindern, dass mit einer gegebenen Emissionsreduktion mehrfach Emissionen ,kompensiert“ werden. Alternativ stellen einige Anbieter eine Spendenbescheinigung aus. Die Option der Zertifikate mit anschließender Stilllegung ist jedoch die bessere Alternative, da nur so das Qualitätssicherungsverfahren nachvollzogen werden kann. Spendenbescheinigungen stellen keinen adäquaten Ersatz dar, können als Ergänzung zur Ausgabe von Zertifikaten aber ein zusätzlicher Bonus sein.

\section{- Die feinen Unterschiede}

Die Kriterien der Anbieter für die Messung des Klimanutzens eines Projekts unterscheiden sich zum Teil erheblich. Mehrere Anbieter führen ihre Projekte im Rahmen des so genannten Clean Development Mechanism (CDM) durch. Der CDM ist ein Element des kürzlich in Kraft getretenen Kyoto-Protokolls. Er erlaubt es den Industriestaaten, Projekte in südlichen Ländern durchzuführen und sich deren Klimanutzen auf ihre KyotoEmissionsziele anzurechnen. Der CDM wird also durch staatliche und zwischenstaatliche Institutionen eingerichtet und überwacht, wodurch ein Mindestmaß an Qualität garantiert wird.

Dies betrifft insbesondere die so genannte Zusätzlichkeit von Projekten. Um den Klimanutzen eines Projekts zu ermitteln, wird ein Referenzszenario erstellt, das wiedergibt, welche Investition oder Wirtschaftsweise in der Abwesenheit des Projekts durchgeführt worden wäre. Dabei muss gezeigt werden, dass das Projekt nicht gleich dem Referenzszenario ist, also ohnehin durchgeführt wor- 
den wäre, sondern eine zusätzliche Anstrengung darstellt. Wenn Zertifikate für ein Projekt ausgestellt werden, das ohnehin durchgeführt worden wäre, steht diesen keine tatsächliche Emissionsreduktion gegenüber, so dass die Emissionen des Kunden letztendlich nicht kompensiert werden.

Bei einigen Anbietern entsprechen die Projekte zusätzlich dem auf Initiative des World Wide Fund for Nature (WWF) von einem internationalen Expertenkreis entwickelten „CDM Gold Standard“. Dies ist ein Set von Qualitätskriterien, das die des CDM noch erweitert. Dies betrifft insbesondere erweiterte Kriterien für die Messung des Entwicklungsnutzens eines Projekts, etwa in Bezug auf die Schaffung von Arbeitsplätzen oder die Förderung benachteiligter Gruppen, sowie detaillierte Bestimmungen für die Einbeziehung der von einem Projekt betroffenen Bevölkerung in die Planungen. Demgegenüber prüfen andere Anbieter ihre Projekte an Hand selbst entwickelter Kriterien. Das muss nicht unbedingt heißen, dass diese Standards schlechter sind. Vielfach sind sie an die offiziellen CDM-Regeln angelehnt und gehen bei manchen Anbietern in ausgewählten Punkten sogar noch darüber hinaus. Aber für den Konsumenten ist es bei diesen Projekten schwerer nachvollziehbar, ob die versprochene Qualität tatsächlich gewährleistet wird. In jedem Fall muss aber sichergestellt sein, dass die behauptete Emissionsreduktion von einer qualifizierten und unabhängigen Partei überprüft wird.
Unterschiede gibt es auch bei den Kompensationsanbietern selber. Im Sinne des Klimaschutzes kann man erwarten, dass ein Anbieter umfassende Informationen über das Klimaproblem zur Verfügung stellt und deutlich macht, dass Kompensation gegenüber der Vermeidung der eigenen Emissionen in jedem Fall die schlechtere Alternative ist. Auch sollte er transparent machen, wie das Geld der Kunden verwendet wird, das heißt welcher Teil davon tatsächlich in die Finanzierung der Projekte fließt und wie die Mittelverwendung kontrolliert wird.

\section{Kompensation für alle?}

Unternehmen, Organisationen und Regierungen machen sich den Kompensationsansatz vermehrt zu Nutze, um ihr Engagement für den Klimaschutz $\mathrm{zu}$ demonstrieren. So werden beispielsweise die gesamten Treibhausgasemissionen der Konferenz für Erneuerbare Energien in Bonn im Juni 2004 von der Bundesregierung kompensiert. Ein anderes Beispiel ist die Fußballweltmeisterschaft in Deutschland 2006, die ebenfalls ,klimaneutral“ gestaltet werden soll.

Die Weiterentwicklung des freiwilligen Zertifikatemarkts hängt nicht zuletzt davon ab, wie die Sensibilisierung der Öffentlichkeit hinsichtlich des Klimawandels fortschreitet. Derzeit kommen nahe$\mathrm{zu}$ ständig Meldungen von Unternehmen und anderen Institutionen, dass sie nun auch ihre Emissionen kompensieren wollen. Dieser Trend wird nur anhalten, wenn damit ein entsprechender Marketingnutzen verbunden ist. Wünschenswert wäre eine Verbesserung der Transparenz auf dem Markt, so dass die Kunden hochwertige von schlechten Angeboten unterscheiden können, etwa durch ein Label. Letztendlich ist aus Klimaschutzsicht aber zu hoffen, dass die Aufmerksamkeitssteigerung für das Klimathema, die durch die Kompensation und die damit verbundenen Marketingmaßnahmen erzielt wird, auch dazu führen, dass sich die Akzeptanz für verbindliche Klimaschutzinstrumente verbessert.

\section{Anmerkung}

(1) Der Artikel ist die gekürzte Version von Sterk, W./ Bunse, M.: Voluntary Compensation of Greenhouse Gas Emissions. Wuppertal 2004, zu beziehen unter www.wupperinst.org oder per E-Mail.

\section{Die Autorlnnen}

Wolfgang Sterk ist wissenschaftlicher Mitarbeiter am Wuppertal Institut für Klima, Umwelt, Energie. Maike Bunse ist dort wissenschaftliche Hilfskraft und Consultant bei triple innova $\mathrm{GmbH}$.

Kontakt: Wuppertal Institut, PF 1004 80, 42004 Wuppertal. Tel. 0202-2492149, E-Mail: wolfgang.sterk@wupperinst.org, maike.bunse@wupperinst.org 
(c) 20I0 Authors; licensee IÖW and oekom verlag. This is an article distributed under the terms of the Creative Commons Attribution Non-Commercial No Derivates License (http://creativecommons.org/licenses/by-nc-nd/3.o/), which permits unrestricted use, distribution, and reproduction in any medium, provided the original work is properly cited. 